\title{
Catálogo de los helmintos parásitos de Costa Rica*
}

\author{
por

\section{Rodrigo Ramón Brenes}

(Recibido para su publicación el 13 de abril de 1961)

Por considerarlo de gran interés, nos hemos propuesto elaborar un catálogo de los helmintos parásitos de este país registrados y estudiados hasta el presente. Al revisar lo referente a helmintos (simple cita, descripción, redescripción, huésped, localización, distribución geográfica) debemos admitir que, al igual que en otros países, en Costa Rica el estudio de la helmintología recibió en un principio poca atención. Además, es necesario aclarar que los aportes publicados por determinados autores nacionales contienen errores o imprecisiones de diversa índole que restan validez a lo publicado.

Para el estudio presente, consultamos en primer término los trabajos que comprenden descripción, redescripción y taxonomía de los helmintos; en segundo lugar, los que dan una simple cita de incidencia parasitaria, basada, en la mayoría de las veces, en el hallazgo de los huevecillos en las materias fecales, especialmente de animales domésticos. En los casos humanos, con excepción de unas pocas publicaciones, omitimos de la bibliografía, por la índole de este trabajo, todos los aportes de carácter esencialmente médico o de salud pública.

Finalmente, en cuanto a la proposición de nuevos nombres ordinales, hacemos eco a los conceptos de BERG (citado por HuBBS, 41), y aceptamos la desinencia (i)formes propuesta por él y posteriormente adoptada por Travassos (87) para nemátodos, gordiáceos, tremátodos, céstodos y acantocéfalos, y por Caballero y Díaz-Ungría (18) para tremátodos digéneos de Venezuela, aunque siguieron en lo demás la nomenclatura propuesta por LA RUE (49).

Para la ordenación taxonómica de los diferentes grupos, corregimos la nomenclatura de acuerdo con las nuevas corrientes establecidas. Así, para tremátodos monogéneos, hẹmos consultado los trabajos de ByCHOWSKy (14), CHAUHAN (24), Hargis (40), Palombi (58) y Sproston (83). En cuanto a tremátodos monogéneos, además de adoptar el sistema de LA RUE (49), revisamos las obras

* Trabajo presentado al Primer Congreso Latinoamericano de Microbiología, en la Ciudad Universitaria, México, D. F., en octubre de 1958, e iniciado durante nuestra estadía como investigador huésped en el Instituto de Biologia de la U.N.A.M.

* Departamento de Parasitología, Facultad de Microbiología, Universidad de Costa Rica. 
de Dawes (31) y Skrjabin (64, 65, 66, 69, 72, 77, 78, 81). Para el ordenamiento de los céstodos se consultó a FuHrmann $(38,39)$, Joyeux y BaER (48), Neveu-Lemaire (57), Spassky (82), Wardle y McLeod (94). En cuanto a los nemátodos, consultamos las obras de BAYLis y DaubneY, (10), Chitwood (30), Morgan y Hawkins (56), Neveu-Lemaire (57), SkRJABIN $(67,68,70,71,73,74,75,76,79,80)$, STILES y Hassall (84), Threkeld (85), Travassos (86) y Yorke y Maplestone (96). Por último, para los acantocéfalos seguimos, en líneas generales, las normas de VAN CLEAVE (88) y Petrotschenko $(59,60)$.

\section{NÖMINA DE HELMINTOS}

\section{CON SUS HUÉSPEDES, LOCALIZACIÓN Y DISTRIBUCIÓN GEOGRÁFICA*}

Para este propósito hemos tomado como guía el trabajo de CASTRo y TrenCHI (22). Algunas veces se observa que el autor de la publicación no citó en su aporte la localización del parásito, ni el nombre científico del huésped o huéspedes en que lo encontró, apareciendo por este motivo únicamente su nombre vulgar. En algunos de estos casos y a sugerencia nuestra, el ingeniero agrónomo Alfonso Jiménez M. del Museo Nacional, tuvo la fineza de darnos con reserva los probables nombres científicos, basados exclusivamente en el vocablo popular mencionado. La cita o citas bibliográficas que se han colocado al lado del nombre de los parásitos, representan las referencias que están en la bibliografía en la presente publicación. Por lo que expusimos anteriormente, en todos los casos en que exista duda del hallazgo del parásito con base en su errada descripción, o a lo equívoco del huésped, a su localización incierta y otras consideraciones, nos hemos permitido colocar después de la cita correspondiente, un signo de interrogación. Consignamos en cada caso las localidades costarricenses de donde se registró la presencia de los helmintos, con todo el detalle dado por los autores respectivos.

\section{Phylum - PLATYHELMINTHES Gegenbaur, 1859 Clase - TREMATODA Rudolphi, 1808 Subclase - MONOGENEA Carus, 1863} Orden - MONOPISTHOCOTYLIFORMES n. nov. (= MONOPISTHOCOTYLEA Odhner, 1919) Suborden - DACTYLOGYRINEA Bychowsky, 1937 Familia - DACTYLOGYRIDAE Bychowsky, 1933

\section{Cleidodiscus travassosi Price, 1938 (61)}

H. D.: Rhamdia rogersi (Regan): branquias.

SAN José: San Pedro de Montes de Oca.

* Abreviaturas utilizadas en el presente catálogo: H. D.: huésped definitivo; H. I.: huésped intermediario; H.: huésped o huéspedes; F, L.: forma larval; F. A.: forma adulta. 
Cleidodiscus chavarriai Price, 1938 (61)

H. D.: Rhamdia rogersi (Regan): branquias.

San Josḱ: San Pedro de Montes de Oca.

Orden - POLYOPISTHOCOTYLIFORMES n. nov.

$(=$ POLYOPISTHOCOTYLEA Odhner, 1912)

Suborden - DISCOCOTYLINEA Bychowsky, 1957

Familia - DISCOCOTYLIDAE Price, 1936

Protomicrocotyle pacifica Meserve, 1938 (53)

H. D.: Xurel marginatus (Hill): branquias.

Guanacaste: Puerto Culebra

Tagia ecuadori (Meserve, 1938) Sproston, 1946 (17)

H. D.: Sphoeroides sp.: branquias

Puntarenas: Mata de Limón.

Subclase-DIGENEA Carus, 1863

Superorden-ANEPITHELIOCYSTIDIA La Rue, 1957

Orden-STRIGEATOIDEA La Rue, 1926

Suborden-STRIGEATA La Rue, 1926

Superfamilia-STRIGEOIDEA Railliet, 1919

Familia-DIPLOSTOMATIDAE Poirier, 1886

Alaria nasuae La Rue y Townsend, 1927 (90(?), 92(?))

H. D.: Coyote (sic.) (=Canis latrans dickeyi Nelson): intestino.

Costa Rica.

Familia-PROTERODIPLOSTOMATIDAE Dubois, 1937

Proterodiplostomum medusae (Dubois, 1936) Caballero, Hidalgo y Grocott, 1957 (18).

H.: Crocodylus acutus acutus Cuvier: intestino delgado.

Ala juela: Los Chiles de Grecia.

Orden-ECHINOSTOMIFORMES Caballero y Díaz-Ungría, 1958

Suborden-ECHINOSTOMATA Szidat, 1939

Superfamilia-ECHINOSTOMATOIDEA Faust, 1929

Familia-COTYLOTRETIDAE Travassos, 1922

*Cotylotretus grandis (Rudophi, 1819) Odhner, 1902 (18)

H.: Ajaia ajaja (L.): intestino delgado.

Puntarenas: Mata de Limón.

Familia-FASCIOLIDAE Railliet, 1895

Fasciola bepatica L., 1758 (11, 19, 21, 27(?), 29(?), 62(?), 92(?))

* Posición taxonómica propuesta por el Dr. Eduardo Caballero y Caballero, seguiendo el sistema de La Rue. 
H. D.: Bos taurus L., Homo sapiens L.: conductos biliares y errática. SAN JosÉ: San José, Matadero Municipal.

Cartago: San Ramón de Tres Ríos.

\section{Familia-RHOPALIIDAE Looss, 1898}

Rhopalias coronatzes Stiles y Hassall, 1898 (19)

H.: Didelphis marsupialis etensis Allen: intestino delgado.

SAN JosÉ: Bello Horizonte, Escazú.

Rhopalias horridus (Diesing, 1850) Stiles y Hassall, 1898 (19)

H.: Didelphis marsupialis etensis Allen: intestino delgado.

SAN JosÉ: Bello Horizonte, Escazú.

Suborden-PARAMPHISTOMATA Szidat, 1836 Superfamilia_PARAMPHISTOMATOIDEA Stiles y Goldberger, 1910 Familia_PARAMPHISTOMATIDAE Fischoeder, 1901

Paramphistomum cervi (Zeder, 1790) Fischoeder, 1901 (19) H.: Bos taurus L.: rumen.

SAN Josḱ: San José, Matadero Municipal.

Familia-DIPLODISCIDAE Skrjabin, 1949

Megalodiscus temperatus (Stafford, 1905) Harwood, 1932 (10)

H.: Rana pipiens Schreber: intestino grueso y recto.

Cartago: Coris.

Familia-HERONIMIDAE Ward, 1917

Heronimus chelydrae MacCallum 1902 (19)

H.: Kinosternon cruentatum Dumeril y Bibron: pulmones.

SAN José: Santa Ana.

Superorden-EPITHELIOCYSTIDIA La Rue, 1957 Orden-PLAGIORCHIIFORMES Caballero y Díaz-Ungría, 1958

Suborden-PLAGIORCHIATA La Rue, 1957

Superfamilia-PLAGIORCHIOIDEA Dollfus, 1930

Familia-DICROCOELIIDAE Odhner, 1910

Controrchis biliopbilus Price, 1928 (43, 44)

H.: Alouatta palliata palliata (Gray) Goodwin: conductos biliares.

Guanacaste: Tilarán, Cañas.

Lutztrema obliquum (Travassos, 1917) Travassos, 1941 (45)

H.: Gymnostinops montezuma (Lesson) Sclates: conductos biliares.

Cartago: Chitaría, Peralta. 
Zonorchis costaricensis Brenes y Jiménez-Quirós, 1959 (9) H.:Gymnostinops montezuma (Lesson) Sclater: conductos biliares. Cartago: Chitaría, Peralta.

Zonorchis macroovaricus Jiménez-Quirós y Arroyo, 1960 (45)

H.: Pteroglosus t. torquatus (Gmelin) : conductos biliares.

Guanacaste: El Silencio, Tilarán.

Familia-LECITHODENDRIIDAE Odhner, 1910

Langeronia macrocirra Caballero y Bravo-Hollis, 1949 (4)

H.: Bufo marinus marinus (L.); Rana warchewitscbii (Schmidt) Dunn.: intestino.

Cartago: Coris.

Ochoterenatrema costaricensis Caballero y Brenes, 1957 (17)

H.: Eptesicus propinquus Peters: intestino delgado.

Heredia: Finca Martínez, Monte de la Cruz.

Prosthodendrium (Prosthodendrium) cordiforme (Braun, 1900) (17)

H.: Eptesicus propinquus Peters: intestino delgado.

Heredia: Finca Martínez, Monte de la Cruz.

Familia-MESOCOELIIDAE Dollfus, 1933

* Mesocoelium travassosi Pereira y Cuocolo, 1940 (18)

H.: Bufo marinus marinus (L.): intestino delgado.

Gunnacaste: Hacienda Lombardía, Tilarán.

Familia-MICROPHALLIDAE Travassos, 1921

Carneophallus skryabini Caballero, 1958 (16)

H.: Didelphis marsupialis etensis Allen: intestino delgado.

SAN José: Bello Horizonte, Escazú.

Familia-OCHETOSOMATIDAE Leăo, 1945

Ochetosoma bravoi Brenes y Arroyo, 1960 (3)

H.: Colubridae, no identificado: esófago.

SAN JosÉ: Escazú.

Ochetosoma miladelarocar Caballero y Vogelsang, 1947 (4)

H.: Drymobius margaritiferus margaritiferus (Schlegel): esófago.

Cartago: Coris.

Familia-PLAGIORCHIIDAE Lühe, 1901

Choledocystus intermedius Caballero, Bravo y Zerecero, 1944 (19)

* Posición taxonómica propuesta por el Dr. Eduardo Caballero, siguiendo el sistema de La Rue, 
H.: Bufo marinus marimus (L.): hígado.

SAN José: Santa Ana.

Glypthelmins facioi Brenes, Jiménez-Quirós, Arroyo y Delgado, 1959 (10)

H.: Rana pipiens (Schreber): intestino delgado.

Cartago: Coris.

Glypthelmins palmipedis (Lutz, 1928) Travassos, 1930 (3)

H.: Bufo marinus marinus (L.): intestino delgado.

Puntarenas: Chapernal.

Parallopharynx gonzalezi Brenes, Arroyo y Montero-Gei, 1960 (5) H.: Basiliscus sp. y Ctenosaurus similis similis Gray: intestino delgado. Alajuela: Río Grande, Atenas.

Plagiorchis Lühe, 1899 (90(?), 92(?))

H.: Tortuga, anfibios, reptiles y algunos pájaros (sic.): intestino. Costa Rica.

*Telorchis Lühe, 1899 (92(?))

H.: Tortuga (sic.): intestino.

Costa Rica.

Telorchis pseudoaculeatus Dollfus, 1929 (17)

H.: Kinosternon cruentatum Dumeril y Bibron: intestino delgado. SAN José: Santa Ana.

Familia_CEPHALOGONIMIDAE Nicoll, 1915

Cephalogonimus americanus Stafford, 1902 (10)

H.: Rana pipiens Schreber: intestino delgado.

Cartago: Coris.

Familia-UROTREMATIDAE Poche, 1926

Urotrema scabridum Braun, 1900 (19)

H.: Eptesicus propinquus Peters: intestino delgado.

Heredia: Finca Martínez, Monte de la Cruz.

Superfamilia-ALLOCREADIOIDEA Nicoll, 1934

Familia-ALLOCREADIIDAE Stossich, 1903

Diploproctodaeum plicitum (Linstow, 1928) Sogandares-Bernal y Hutton, 1958 (17)

* Creemos que tal vez este tremátodo señalado por Víquez sea Telorchis pseudoasu. leatus Dollfus, 1929, encontrado y redescrito en 1958. 
H.: Sphoeroides sp.: Intestino delgado.

Puntarenas: Mata de Limón.

Helicometra sinuata (Rudolphi, 1819)

$\mathrm{H}$ ::Muraena sp.: intestino.

Guanacaste: Puerto Culebra.

Familia-MONORCHIIDAE Odhner, 1911

Tergestia laticollis (Rudolphi, 1819) (51)

H.: Caranx caballus Günther: intestino.

Guanacaste: Puerto Culebra.

Proctotrema costaricae Manter, 1940 (51)

H.: Pez gruñidor, de líneas amarillas, posiblemente del género Medialuna: intestino y ciego.

Guanacaste: Puerto Culebra.

Familia_GORGODERIDAE Looss, 1901

Gorgoderind (Gorgoderina) megalorchis Bravo-Hollis, 1948 (19)

H.: Bufo marinus marinus (L.): vejiga urinaria.

SAN José: Santa Ana.

Familia-TROGLOTREMATIDAE, Odhner, 1914

Paragonimus rudis (Diesing, 1850) Braun, 1899 (15)

H.:Urocyon cintreoargenteus costaricensis Goodwin: pulmón. Ala juela: Carrizal.

Familia-HETEROPHYIDAE Odhner, 1914

Eurybelmis costaricensis Brenes, Arroyo y Jiménez-Quirós, 1960 (6)

H.: Mustela frenata costaricensis Goldman: intestino delgado.

Cartago: El Alto, Tres Ríos.

Orden - OPISTHORCHIIFORMES Caballero y Díaz-Ungría, 1958 Suborden - OPISTHORCHIATA La Rue, 1957 Superfamilia - OPISTHORCHIOIDEA Faust, 1929 Familia - ACANTHOSTOMATIDAE Poche, 1926

Acanthostomum acuti Caballero y Brenes, 1959 (18) H.:Crocodylus acutus acutus Cuvier: intestino delgado. Alajuela: Los Chiles de Grecia. 
SAN José: Río Tiribí, Paso Ancho.

Clase - CESTODA (Van Beneden, 1849) Monticelli, 1892, emend., Fuhrmann, 1931

Orden - PROTEOCEPHALIFORMES n. nov. (= PROTEOCEPHALA Wardle y McLeod, 1952)

\section{Familia — PROTEOCEPHALIDAE La Rue, 1914}

Opbiotaenia La Rue, 1911 (90(?), 92(?))

$\mathrm{H}$.:Constrictor constrictor imperator (Daudin): intestino.

Costa Rica.

Orden - CYCLOPHYLLIIFORMES n. nov. (= CYCLOPHYLLIDEA Braun, 1900)

Familia - ANOPLOCEPHALIDAE Cholodkowsky, 1902

* Avitellina centripunctata (Rivolta, 1874) (91(?), 92(?))

$\mathrm{H}$. :Canis familiaris L.: únicamente huevecillos y anillos en las heces.

Costa Rica.

Anoplocephala magna (Abildgaard, 1789) Sprengel, 1905 (26(?), 29(?)

H.: Equus caballus L.: intestino.

zosta Rica.

Anoplocephala perfoliata (Goeze, 1782) Blanchard, 1848 (26 (?), 29(?), 52(?))

H.: Equus caballus L.; Equus caballus $\times$ Equus asinus: última porción del intestino delgado y ciego, rara vez en el colon.

Limón.

Moniezia (Blanchariezia) benedeni (Moniez, 1879) Blanchard, 1891 (27(?), 29(?), 37, 92(?))

H.: Bos taurus L.; Bradypus griseus griseus (Gray): intestino.

Cartago: Turrialba.

Moniezia (Moniezia) expansa (Rudolphi, 1810) Blanchard, 1891 27(?), 29(?), 91(?), 92(?)

H.: Bos taurus L.: intestino delgado.

Costa Rica.

Oochoristica pennsylvanica Chandler y Melvin, 1951 (36)

H.:Didelphis marsupialis etensis Allen: intestino delgado.

SAN JosÉ: Bello Horizonte, Escazú.

* Opinamos que se trata de otro céstodo, porque nunca se ha seportado la presencia de este género en Canidae. 
Paranoplocephala mamillana (Mehlis, 1831) Baer, 1927 26(?), 29(?)). H.: Equus caballus L.: intestino delgado.

Costa Rica.

\section{Familia - TAENIIDAE Ludwig, 1886}

Taenia laticollis Rudolphi, 1819 (37) delgado.

H.: Urocyon cinereoargenteus costaricensis Goodwin: F. A.: intestino Ala juela: Carrizal.

Taenia saginata Goeze, 1782 (47, 62(?), 90(?), 92(?))

H. D.: Homo sapiens L.: F. A.: intestino delgado.

H. I.: Bos taurus L.: F. L.: Cysticercus bovis: músculos internos y externos de las quijadas, en el corazón y músculos inguinales; no tan frecuente en la lengua, músculos del esófago, cuello y pecho, raramente en las vísceras.

Cartago: San Rafael de Oreamuno; Guanacaste.

Taenia solium L., 1758 (25(?), 29(?), 47, 90(?), 92(?))

H. D.: Homo sapiens L.: F. A.: intestino delgado.

H. I.: Sus scrofa L.: F. L.: Cysticercus cellulosae musculatura (triceps subcapilaris, pectoralis, intercostales, cervicalis, diafragma, contractores de las extremidades, lengua y corazón). Las partes más frecuentes son: la región maxilar, axilar e inguinal. Además se le puede encontrar en el cerebro, ojos, hígado, bazo, pulmón, glándulas linfáticas y grasa.

Costa Rica.

Taenia taeniaeformis (Batsch, 1786) Wolffhügel, 1911 (36, 37, 55, 93)

H. D.: Felis domestica L., Felis wiedii nicaraguae (J. A. Allen), F. A.: intestino delgado.

H. I.: Rattus norvegicus norvegicus (Berkenhout), F. L.: hígado.

San José, Puntarenas: Mata de Limón.

\section{Familia - DAVAINEIDAE Fuhrmann, 1907}

Davainea proglottina (Davaine, 1869) (28(?), 29(?))

H.: Gallus gallus domesticus L.: intestino delgado.

Costa Rica.

Raillietina (Raillietina) ecbinobotbrida (Megnin, 1881) (28(?), 29(?), 90(?), $92($ ?))

H.: Gallus gallus domesticus L.: intestino delgado.

Costa Rica.

Raillietina (Raillietina) tetragona (Molin, 1858) (28(?), 29(?), 35, 90(?), 92(?))

H.: Gallus gallus domesticus L.: intestino delgado. 
Costa Rica.

Familia - HYMENOLEPIDIDAE Railliet y Henry, 1909

Diorchis acuininata (Clerc, 1902) (92(?))

H.: ?: intestino delgado.

Costa Rica.

Haploparaxis Clerc, 1903 (92(?)) (sic.):?

H.: (Turdus grayz casius) (Bonaparte) "yigüirro y otros pájaros"

Costa Rica.

Hymenolepis cantaniana Polonio, 1860 (91(?), 92(?))

H.: ? ?

Costa Rica.

Hymenolepis diminuta (Rudolphi, 1819) Blanchard, 1891 (34, 47, 91(?), 92(?), 93)

H.: Homo sapiens L.; Rattus norvegicus norvegicus (Berkenhout): intestino delgado.

Costa Rica.

*Hymenolepis nana (v. Siebold, 1852) Blanchard, 1891 (47, 91(?), $92(?), 93)$

H.:Homo sapiens L.; Rattus norvegicus norvegicus (Berkenhout): intestino delgado.

Costa Rica.

Familia - DILEPIDIDAE Fuhrmann, 1907

Amoebotaenia Cohn, 1900 (91(?), 92(?))

H.:Gallus gallus domesticus L.: intestino delgado.

Costa Rica.

Amoebotaenia spibenoides Railliet, 1892 (28(?), 29(?))

H.:Gallus gallus domesticues L.: intestino delgado.

Costa Rica.

Anomotaenia Cohn, 1900 (92(?))

H.: "Yigüirro" (sic.): (=Turdus gragi casius Bonaparte): se encontró casualmente en el "estiércol" del huésped.

Costa Rica.

* Dudamos del hallazgo de este parásito por Víquez en Turdus grayi casius y Bothrops atrox atrox. 
Dipylidium caninum (L., 1758) (33, 91(?), 92(?)) delgado.

H.: Homo sapiens L., Canis familiaris L. Felis domestica L.: intestino

Costa Rica.

Choanotaenia Railliet, 1896 (91(?), 92(?))

H.: Gallus gallus domesticus L.: intestino delgado.

Costa Rica.

Choanotaenia infundibulum Bloch, 1779 (28(?), 29(?))

H.: Gallus gallus domesticus L.: intestino delgado.

Costa Rica.

Phylum-NEMATODA (Rudolphi, 1808) Cobb, 1919

Clase-SECERNENTEA (v Linstow, 1905) Dougherty, 1958

Orden-RHABDITIIFORMES n. nov. (= RHABDITIDA Chitwood, 1933)

Suborden-RHABDITINA Chitwood, 1950

Superfamilia-RHABDITOIDEA Travassos, 1920

Familia - RHABDIASIDAE Railliet, 1915

Rhabdias sphaerocepbala Goodey, 1924 (7)

H.:Bufo marinus marinus (L.): pulmón.

Alajuela: La Peña, Zarcero. San José: Río Tiribí, Paso Ancho.

Familia - STRONGYLOIDIDAE Chitwood y McIntosh, 1934

Strongyloides ratti Sanground, 1925 (93)

H.: Rattus norvegicus norvegicus (Berkenhout): primera porción del intestino delgado.

SAN JosÉ: San José.

Strongyloides stercoralis (Bavay, 1876) (47, 91(?))

H.: Homo sapiens L.: intestino delgado.

Costa Rica.

Suborden - STRONGYLINA Railliet y Henry, 1913

Superfamilia - STRONGYLOIDEA Weinland, 1858

Familia - ANCYLOSTOMATIDAE Looss, 1905

Ancylostoma braziliense Gómes de Faria, 1910 (91(?), 92(?))

H.: Potos flavus arborensis Goodwin: intestino.

Costa Rica.

Ancylostoma caninum (Ercolani, 1859) Linstow, 1889 (90(?), 92(?))

H.: Canis latrans dickeyi Nelson; Canis familiaris L.; Felis domestica L.: intestino delgado.

Costa Rica. 
Ancylostoma duodenale (Dubini, 1843) Creplin, 1845 (47, 63, 90(?), $92(?))$

H.: Homo sapiens L.: intestino delgado.

Costa Rica.

Bunostomum pblebotomum (Railliet, 1900) Railliet, 1902 (12(?), $27(?), 29(?))$

H.: Bos taurus L.: intestino delgado, principalmente duodeno.

Cartago: Turrialba. SAN José: San Isidro de Coronado.

Bunostomum trigonocephalum Rodolphi, 1908 (54(?))

H.: Capra bircus L.: intestino delgado.

SAN José: Sabanilla de Montes de Oca.

Gaigeria pacbycelis Railliet y Henry, 1910 (12(?), 54(?))

H.: Bos taurus L.; Capra bircus L.: intestino delgado.

Cartago: Turrialba. SAN José: Sabanilla de Montes de Oca.

Necator americanus Stiles, 1902 (47, 63)

H.: Homo sapiens L.: intestino delgado.

Costa Rica.

\section{Familia - DIAPHANOCEPHALIDAE Travassos, 1919}

Kalicepbalus macrovulvus Caballero, 1954 (8)

H.: Botbrops atrox atrox (L.): intestino delgado.

SAN José: San José, Hospital San Juan de Dios.

\section{Familia - STRONGYLIDAE Baird, 1953}

Alfortia edentatus (Looss, 1900) Skrjabin, 1933 (26(?), 29(?), 52(?)) H.: Equus caballus L.; Equus caballus $\times$ Equus asinus: ciego y colon. Costa Rica.

Delafondia vulgaris (Looss, 1900) Skrjabin, 1933 (26(?), 29(?), 52(?)) H.: Equus caballus L.; Equus caballus $\times$ Equus asinus: ciego y colon. LIMÓN.

Globocephalus urosubulatus (Alessandrini, 1909) (25(?), 29(?)) H.: Sus scrofa L.: intestino delgado.

SAN José: Santa Ana; Puriscal.

Strongylus equinus Müller, 1780 (26(?), 29(?), 52(?))

H.: Equus caballus L.; Equus caballus $\times$ Equus asinus: ciego y colon. LIMÓN.

Triodontophorus tenuicollis Baulenger, 1916 (26(?), 29(?), 52(?)) H.: Equus caballus L.; Equus caballus $\times$ Equas asinus: ciego y colon. 


\section{I.IMÓN.}

Familia - STEPHANURIDAE Travassos y Vogelsang, 1933

Stephanurus dentatus Diesing, 1839 (25(?), 29(?), 62(?))

H.: Sus scrofa L.; Bos taurus L.: riñón e hígado.

Costa Rica.

\section{Familia - SYNGAMIDAE Leiper, 1912}

Syngamus (Syngamus) trachea (Montagu, 1811), Chapin, 1925 (29(?))

H.: Gallus gallus domesticus L.; Meleagris gallopavo L.: tráquea.

Costa Rica.

Familia - TRICHONEMATIDAE Witenberg, 1925

Cylicocyclus insignt (Boulenger, 1917) (52(?))

H.: Equus caballus L.: ciego y colon.

LIMÓN.

Oesophagostomum Molin, 1861 (54(?))

H.: Capra bircus L.: intestino grueso.

SAN Josḱ: Sabanilla de Montes de Oca.

Oesophagostomum (Oesophagostomurn) dentatum (Rudolphi, 1803) Molin, 1861 (25(?), 29(?))

H.: Sus scrofa L.: intestino grueso.

Costa Rica.

Oesophagostomum (Bosicola) radiatum (Rudolphi, 1803) Railliet, 1893 (12(?), 27(?), 29(?))

H.:Bos taurus L.: nódulos del intestino y colon.

Cartago: Turrialba.

*Oesophagostomum (Hysteracrum) venulosum (Rudolphi, 1809) Railliet y Henry, 1913 (91(?), 92(?))

H.: Rata, cabros y ovejas (sic.): ?

Costa Rica.

\section{Superfamilia - TRICHOSTRONGYLOIDEA Cram, 1927 \\ Familia - TRICHOSTRONGYLIDAE Leiper, 1912}

Cooperia Ransom, 1907 (12(?))

H.: Bos tarrus L.: nódulos del intestino y colon.

Cartago: Turrialba.

Haemonchus contortus (Rudolphi, 1803) Cobb, 1898 (12(?), 27(?),

* Nunca ha sido señalado como parásito de roedores. 
29(?), 54(?))

H.: Bos taurus L.; Capra bircus L.: abomasum.

Cartago: Turrialba. San José: Sabanilla de Montes de Oca.

Mecistocirrus digitatus (v. Linstow, 1906) Railliet y Henry, 1912 (12(?), 27(?), 29(?), 32, 91(?), 92(?))

H.: Bos taurus L.: intestino delgado, abomasum.

Cartago: Turrialba.

*Nematodirus Ransom, 1907 (52(?))

H.: Equus caballus L.; Equus caballus $\times$ Equus asinus:?

LIMÓN.

Ostertagia Ransom, 1907 (12(?), 54(?))

H.: Bos taurus L.; Capra bircus L.: intestino delgado, abomasum,

Cartagio: Turrialba. San José: Sabanilla de Montes de Oca.

Oswaldocruzia subauricularis (Rudolphi, 1819) Travassos, 1917 (7)

H.: Bufo marinus marinus (L.): intestino delgado.

Guanacaste: Tilarán. San José: Paso Ancho.

Trichostrongylus Looss, 1905 (12(?))

H.: Bos taurus L.: ?

Cartago: Turrialba.

\section{Familia - HELIGMOSOMATIDAE Cram, 1927}

Nippostrongylus braziliensis (Travassos, 1914) Travassos y Darriba, 1929 (93)

H.: Rattus norvegicus norvegicus (Berkenhout): intestino. Costa Rica.

Superfamilia - METASTRONGYLOIDEA Lane, 1917

Familia - METASTRONGYLIDAE Leiper, 1909

Metastrongylus elongatus (Dujardin, 1845) Railliet y Henry, 1911 (25(?), 29(?), 62(?))

H.: Sus scrofa L.: bronquios y tráquea.

Costa Rica.

Familia - DICTYOCAULIDAE Skrjabin, 1941

Dictyocaulus Railliet y Henry, 1907 (54(?))

H.: Capra bircus L.: aparato respiratorio.

San José: Sabanilla de Montes de Oca.

* Nunca ha sido señalado como parásito de equinos 
Dictyocaulus (Dictyocaulus) arnfieldi (Cobb., 1884) Railliet y Henry, 1907 (26(?), 29(?), 52(?))

H.:Equus caballus L.; Equus caballus $\times$ Eques asinus: pulmón y bronquios.

Limón.

Dictyocaulus (Micrurocaulus) eckerti Skrjabin, 1931 (12(?), 27(?), 29(?), 92(?))

H.: Bos taurus L.: pulmón.

CARTAGo: Turrialba.

Suborden - ASCARIDINA Skrjabin, 1915

Superfamilia - OXYUROIDEA Railliet, 1916

Familia - AORURIDAE Skrjabin y Schikhobalova, 1951

Leidynema appendiculata (Leidy, 1850) (89)

H.: Blatta orientalis L.; Periplaneta australasiae (Fabr.): intestino posterios.

Costa Rica.

Familia - ATRACTIDAE Travassos, 1919

Atractis caballeroi Brenes y Bravo-Hollis, 1960 (8)

H.: Kinosternon cruentatum Dumeril y Bibron: intestino grueso.

SAN José: Santa Ana.

Atractis impura Caballero, 1944 (8)

H.: Kinosternon cruentatum Dumeril y Bibron: intestino grueso.

SAN José: Santa Ana.

Cyrtosomum longicaudatum Brenes y Bravo-Hollis, 1960 (8)

$\mathrm{H}$.: Ctenosaurus similis sinzilis (Gray): intestino grueso.

Guanacaste: Liberia. Puntarenas: Mata de Limón.

Enterobius vermicularis (L., 1758) (47, 92(?))

H.: Homo sapiens L.: intestino grueso.

Costa Rica.

Oxyuris equi (Schrank, 1788) (26(?), 29(?), 52(?) 91(?), 92(?)) H.: Equus caballus L.; Equus caballus $\times$ Equus asinus: intestino grueso. LiMÓN.

Familia - COSMOCERCIDAE Travassos, 1925

Oxysomatium Railliet y Henry, 1913 (7, 90(?), 92(?))

H.: Culebras, beker y coral (sic.): intestino.

Costa Rica. 
Oxysomatium itzocanensis (Bravo, 1943) Skrjabin, 1951 (7)

H.: Bufo marinus marinus L.: intestino delgado.

SAN José: San José.

Familia - HETEROXYNEMATIDAE Skrjabin y Shikhobalova, 1948

Aspicularis tetraptera Nitzch, 1821 (93)

H.: Rattus rattus (Erxleben): intestino delgado.

SAN JosÉ: Escazú.

\section{Familia - OXYURIDAE Gobbold, 1864}

Ozolaimus cirratus (Linstow, 1906) Railliet y Henry, 1912 (2)

H.: Ctenosaurus similis similis (Gray): intestino grueso.

Guanacaste: Liberia. Puntarenas: Mata de Limón.

Ozolaimus megatyphlon (Rudolphi, 1819) Dujardin, 1845 (2)

H.: Ctenosaurus similis similis (Gray): intestino grueso.

Guanacaste: Liberia. Puntarenas: Mata de Limón.

Superfamilia - ASCAROIDEA Railliet y Henry, 1915

Familia - ANISAKIDAE Skrjabin y Karokhin, 1945

Neoascaris vitulorum (Goeze, 1782) Travassos, 1927 (12(?), 27(?), $29(?))$

H.: Bos taurus L.: intestino delgado.

Cartago: Turrialba.

Porrocaecum (Porrocaecum) angusticolle (Molin, 1860) Baylis y Daubney, 1922 (90(?), 92(?))

H.: Carraco (sic.): intestino delgado.

Costa Rica.

Toxocara Stiles, 1905 (91(?), 92(?))

H.: Canis familiaris L.; Felis domestica L.: intestino delgado.

Costa Rica.

Toxocara canis (Werner, 1782) Stiles, 1905 (92(?))

H.: Canis familiaris L.: intestino delgado.

Costa Rica.

Toxocara mystax (Zeder, 1800) Stiles, 1900 (92(?))

H.: Felis domestica L.: intestino delgado.

Costa Rica. 
Familia-ASCARIDAE Baird, 1853

Ascaris lumbricoides L., 1758 (23, 47, 91(?), 93)).

H.: Homo sapiens L. Canis familiaris L.: intestino delgado y vías biliares.

Costa Rica.

Ascaris sutum Goeze, 1782 (25(?), 29(?), 62(?))

H.: Sus scrofa L.: conductos biliares y otros órganos.

SAN JosÉ: San José, Matadero Municipal.

Opbidascaris Baylis, 1921 (92(?))

H.: Culebra beker (sic.) (= Constrictor constrictor imperator (Dau$\operatorname{din})):$ ?

Costa Rica.

Parascaris equorum (Goeze, 1782) Yorke y Maplestone, 1926 (26(?), 29(?), 52(?))

H.: Equus caballus L.; Equus caballus $\times$ Equus asinus: intestino delgado. LIMÓN.

Familia - ASCARIDIIDAE Skrjabin y Mosgovoy, 1953

Ascaridia columbae (Gmelin, 1790) Travassos, 1913 (92(?)) intestino.

H.: Paloma collareja (sic.) (= Columba fasciata crissalis Salvadori):

Costa Rica.

Ascaridia galli (Schrank, 1788) Freeborn, 1923 (28(?), 29(?), 91(?), $92(?))$

H.: Gallus gallus domesticus L.; Meleagris gallopavo L. y "patos" (sic.): intestino.

Orden - SPIRURIIFORMES n. nov. (= SPIRURIDA Chitwood, 1933)

Suborden - SPIRURINA Railliet y Henry, 1915

Superfamilia - SPIRUROIDEA Railliet y Henry, 1915

Familia. - GONGYLONEMATIDAE Sobolev, 1949

Gongylonema neoplasticum (Fibiger y Ditlevsen, 1914) Ransom y Hall, $1916(93)$

H.: Rattus norvegicus norvegicus (Berkenhout): esófago.

SAN José: San José, Matadero Municipal.

Familia-PHYSALOPTERIDAE Leiper, 1908

Pbysaloptera Rudolphi, 1819 (91(?), 92(?))

H.: Culebra terciopelo (sic.) (= Botbrops atrox atrox (L.)): ? 
Costa RicA.

\section{Familia_SPIRURIDAE Oerley, 1885}

Habronema megastoma (Rudolphi, 1819) Seurat, 1914 (26(?), 29(?)) H.: Equus caballus L.: ?

Costa Rica.

Habronema microstoma (Schneider, 1866) Rans., 1911 (26(?), 29(?), $52(?))$

H.: Equus caballus L.: estómago.

LıMÓN.

Mastophorus muris (Gmelin, 1790) (93)

H.: Rattus norvegicus norvegicus (Berkenhout): estómago.

Costa Rica.

Spiroxys Schneider, 1866 (92(?)).

H.: Tortuga de tierra (sic.) (= Geomyda manni Dunn): ?

Costa Rica.

\section{Familia - THELAZIIDAE Railliet, 1916}

Leiurus leptocephalus (Rudolphi, 1819) Leuckart, 1850 (42)

H.: Bradypus griseus griseus (Gray, 1871): intestino delgado.

CARTAgo: Turrialba.

Pbysocephalus sexalatus (Molin, 1860) Diesing, 1861 (25(?), 29(?))

H. D.: Sus scrofa L.: F. A.: ?

H. I.: Phanaeus sp.: F. L.: ?

Costa RicA.

*Thelazia Bosc, 1819 (90(?), 92(?))

H.: Culebra beker (sic.) (= Constrictor constrictor imperator (Daudin) ) : estómago.

Costa Rica.

$$
\begin{gathered}
\text { Superfamilia - FILARIODEA Weinland, } 1858 \\
\text { Familia - FILARIIDAE Claus, } 1885
\end{gathered}
$$

Litomosoides peñai Jiménez-Quirós y Arroyo, 1960 (46)

H.: Hemiderma perspicillatum aztecum (Saussure): cavidad peritoneal.

* Nos parece muy raro el hallazgo, porque este género es parásito de los conductos lacrimales, córnea de mamíferos o membrana nictitante de las aves. 
SAN José: Santa Ana.

Ochoterenella digiticauda Caballero, 1944 (7)

H.: Bufo marinus marinus (L.): hojas peritoneales.

SAN José: Santa Ana.

Setaria cervi (Rudolphi, 1819) Baylis, 1936 (91(?), 92(?))

H.: Bos taurus L.: cavidad peritoneal.

Costa Rica.

Setaria equina (Abildaard, 1789) (26(?), 29(?), 52(?), 91(?), $92(?))$.

H.: Equus caballus L.: cavidad abdominal, pleural, peritoneal; ojo y escroto.

LimóN.

Wuchereria bancrofti (Cobbold, 1877) Seurat, 1921 (13, 50).

H.: Homo sapiens L.: vías linfáticas y microfilarias en sangre periférica.

Limón: Puerto Limón, Barrio Roosevelt.

Subclase - ADENOPHORA (v. Linstow, 1905) Chitwood, 1958

Orden - ENOPLIIFORMES n. nov. (= ENOPLIDA Chitwood, 1933)

Suborden - DORYLAMINA Chitwood, 1950

Superfamilia - TRICHOCEPHALOIDEA Spassky, 1954

Familia - CAPILLARIIDAE Neveu-Lemaire, 1936

Capillaria Zeder, 1800 (9, $12($ ?))

H.: Bos taurus L.; Kinosternon cruentatum Dumeril y Bibron: intestino delgado.

SAN JosÉ: Santa Ana.

Capillaris anmulosa (Dujardin, 1845) Travassos, 1915 (29(?))

H.: Aves (sic.): esófago y buche.

Costa Rica.

Capillaria obsignata Madsen, 1945 (91(?), 92(?))

H.: Columba livia L.: ?

Costa Rica.

Familia - TRICHOCEPHALIDAE Baird, 1853

Trichocephalus ovis Abildaard, 1795 (12(?), 54(?), 91(?), 92(?))

H.: Bos taurus L.: intestino grueso.

Cartago: Turrialba. San José: Sabanilla de Montes de Oca.

T'richocephalus suis Schrank, 1788 (25(?), 29(?))

H.: Sus scrola L.: ciego y colon. 
Costa Rica.

I'ricbocephalus trichintus (L., 1758) Blanchard, 1891 (47, 93)

H.: Homo sapiens L.: intestino grueso.

Costa Rica.

Trichocephalus vulpis Froelich, 1789 (91(?), 92(?))

H.: Canis familiaris L.: intestino grueso.

Costa Rica.

Familia - TRICHOSOMOIDIDAE Yorke y Maplestone, 1926

Trichosomoides crassicauda (Bellingham, 1840) Railliet, 1895 (93)

H.: Rattus norvegicus norvegicus (Berkenhout): vejiga urinaria.

SaN José: San José.

Phylum-ACANTHOCEPHALES (Rudolphi, 1808) Skrjabin y Schultz, 1931

Clase-ACANTHOCEPHALA Rudolphi, 1808

Subclase-ECHINORHYNCHINEA Petrotschenko, 1956

Orden-POLYMORPHIDIFORMES n. nov. (= POLYMORPHIDA

Petrotschenko, 1956)

Familia-POLYMORPHIDAE (Meyer, 1951) Petrotschenko, 1958

Hexaglandula corynosoma (Travassos, 1915) Petrotschenko, 1956 (92(?))

H.: Comadreja (sic.) (= Mustela frenata costaricensis Goldman): intestino.

Costa Rica.

*Polymorphus minutus (Goeze, 1782) Lühe, 1911 (91(?), 92(?))

H.: Rata, pájaros y aves semiacuáticas (sic.): ?

Costa Rica.

Subclase - GIGANTORHYNCHINEA Petrotschenko, 1956

Orden - GIGANTORNYNCHIDIFORMES n. nov.

(=GIGANTORHYNCHIDA Southwell et Macfie, 1925)

Familia - GIGANTORHYNCHIDAE (Hamann, 1892) Petrotschenko, 1958

Centrorbynchus tumidulus (Rudolphi, 1819) (92(?))

H.: Rana (sic.): intestino.

Costa Rica.

* No se ha señalado en roedores. Nos parece que lo confundió en ratas con Monili. formis moniliformis. 
Familia-PROSTHORHYNCHIDAE Petrotschenko, 1956

*Prosthorbynchus angrensis Travassos, 1926 (92(?)) intestino.

H.: Comadreja (sic.) (= Mustela frenata costarricensis Goldman) :

Costa Rica.

Orden - OLIGACANTHORHYNCHIDIFORMES n. nov (= OLIGACANTHORHYNCHIDA Petrotschenko, 1956) Familia - MONILIFORMIDAE Van Cleave, 1924

** Moniliformis moniliformis (Bremser, 1819) Travassos, 1915 (89, 91(?), 93)

H. D.: Rattus norvegicus norvegicus (Berkenhout): F. A.:, intestino delgado.

H. I.: Periplaneta anstralasiae (Fabr.): F. L.:, cavidad general.

$$
\text { Familia - PACHYSENTIDAE Meyer, } 1931
$$

Oncicola oncicola (v. Ihering, 1892) Travassos, 1916 (97)

H. I.: Gallus gallus domesticus L.: tejido subcutáneo y muscular de la región pectoral.

Puntarenas: Parrita, Aguirre.

\section{AGRADECIMIENTO}

El autor desea expresar su sincero agradecimiento al Prof. Dr. Eduardo Caballero y C. por su consejo y orientación; a la Srta. Margarita Bravo-Hollis y al Dr. Howard A. Winter $\dagger$, del Instituto de Biología, U. N. A. M., y a sus compañeros Lic. Guido Arroyo S., Dr. Otto Jiménez-Quirós, Dr. Rodrigo Zeledón, y Guillermo Muñoz, de la Facultad de Microbiología, U. C. R., por su colaboración en diversas formas durante la preparación de este trabajo; al Ing. Agr. Alfonso Jiménez M., por la identificación de algunas especies de huéspedes; y finalmente, al Dr. Donald Heyneman, del Departamento de Zoología, U. C. L. A., por su colaboración y su crítica de parte del manuscrito original.

\section{RESUMEN}

Se presenta una lista de los helmintos parásitos de Costa Rica con su

Sólo se ha reportado en pájaros la fase adulta.

** En este caso nos parece que Víquez confundió esta especie con Macracantborbynchus birudinaceus.

$\dagger$ De grata memoria. 
huésped, localización y distribución geográfica, ordenados a la luz de las nuevas corrientes taxonómicas. Se proponen algunos nuevos nombres ordinarios con la aceptación de la desinencia (i)formes para esta categoría.

\section{SUMMARY}

A check list is presented of parasitic helminths of Costa Rica, with indication of host, localization, and geographical distribution, arranged according to recent taxonomic trends. Some ordinal names are amended in order to arrive at the consistent use of the ending (i)formes for this category.

\section{BIBLIOGRAFI.A}

1. BAylis, H. A. y R. Daubney

1926. A Synopsis of the families and genera of Nematoda. xxxvi +277 pp. British Museum. London.

2. Bravo-Hollis, M., y R. R. Brenes

1960. Helmintos de la República de Costa Rica. X. Nematoda 4. Sobre la posición taxonómica de los géneros Ozolaimus Dujardin, 1845 y Macracis Gedoelst, 1916. An. Inst. Biol. Méx., 30 (1-2): 209-225.

3. Brenes, R. R., y G. Arroyo

1960. Helmintos de la República de Costa Rica. XVII. Descripción de Ocbetosoma bravoi n. sp. y redescripción de Glypthelmins palmipedis (Lutz, 1928) Travassos, 1930 Rev. Biol. Trop., 8 (2): 239-245.

4. Brenes, R. R., G. Arroyo y E. Delgado

1959. Helmintos de la República de Costa Rica. XI. Sobre la validez del género Langeronia Caballero y Bravo, 1949. (Trematoda: Lecithodendriidae) y hallazgo de Ochetosoma miladelarocai Caballero y Vogelsang, 1947. Rev. Biol. Trop., 7 (1): 81-87.

5. Brenes, R. R., G. Arroyo y F. Montero-Gei

1960. Helmintos de la República de Costa Rica. XVI. Parallophirrynx gonzalezi n. sp. (Trematoda: Plagiorchidae) Rev. Biol. Trop., 8 (2): 233-237.

6. Brenes, R. R., G. Arroyo y O. Jiménez-Quirós

1960. Helmintos de la República de Costa Rica. XVIII. Una nueva especie de Eurybelmins (Trematoda: Heterophyidae), parásito de Mustela frenata cos. taricensis. Rev: Biol. Trop.; 8 (2): 247.251.

7. Brenes, R. R., y M Bravo-Hollis

1959. Helmintos de la República de Costa Rica. VIII. Nematoda 2. Algunos nemátodos de Bufo marinus marinus (L.) y Algunas consideraciones sobre los géneros Oxysomatium y Aplectana. Rev. Biol. Trop.; 7 (1): 35-55.

8. Brenes, R. R., y M. Bravo-Hollis

1960. Helmintos de la República de Costa Rica. IX. Nematoda 3. Algunos nemátodos de reptiles, con descripción de dos nuevas especies: Atractis caba. 
lleroi n. sp. y Cyrtosomum longicaudatum n. sp. Libro Jubilar Dr. Eduardo Caballero, pp. 451-464.

9. BREnes, R. R., y O. JimÉnez-Quirós

1959. Helmintos de la República de Costa Rica. XII. Zonorcbis costaricensis n. sp. (Trematoda: Dicrocoeliidae) parásito de vías biliares de Gymnostinops montezuma (Icteridae). Rer. Biol. Trop., 7 (1): 125-129.

10. Brenes, R. R., O. Jiménez-Quirós, G. Arroyo y E. Delgado

1959. Helmintos de la República de Costa Rica. XIII. Algunos tremátodos de Rana pipiens. Descripción de Glypthelmins facioi n. sp. Rev. Biol. Troț., 7 (2): 191-197.

11. Brenes, R. R., y J. B. Soto

1953. Contribución al estudio de la Clase Trematoda, con especial referencia a Fasciola bepatica y moluscos bospederos intermediarios de tremátodos en Costa Rica. Tesis de Grado. 95 pp. Universidad de Costa Rica.

12. Briceño CARrillo, A.

1944. "Estudio de los parásitos internos más corrientes en los terneros de la región de Turrialba y su tratamiento. Tesis de Grado. 79 pp. Universidad de Costa Rica.

13. Burrs, D. C. A.

1947. Filarial Infection in Costa Rica. Amer, J. Trop. Med, 27 (5): 607-615.

14. BYCHOWSKY, B. E.

1957. Tremátodos Monogéneos, su sistemática y filogenia. $509 \mathrm{pp}$. Isdatelstv Akademii Nauk SSSR. Moskva. (En ruso).

15. Caballero y C., E.

1957. Presencia de Paragonimus rudis (Diesing, 1850) Braun, 1899 en mamíferos silvestres en Centro América. An. Inst. Biol. Méx., 27 (1-2): 397-401.

16. Caballero y C., E.

1958. Helmintos de la República de Costa Rica. III. Descripción de una nueva especie de tremátodo digéneo Carneophallus skryabini nov. sp. An. Es. Nac. Cien. Biol., 9 (1-4): 77-83.

17. Caballero, y C., E., y R. R. Brenes

1958. Helmintos de la República de Costa Rica. VI. Algunos tremátodos de peces, reptiles y mamíferos. An. Inst. Biol. Méx., 28 (1-2): 217-240.

18. Caballero, y C., E., y R. R. Brenes

1959. Helmintos de la República de Costa Rica. VII. Tremátodos de algunos vertebrados salvajes, con descripción de una nueva especie de Acantbostomum Looss, 1899. An. Inst. Biol. Méx., 29 (1-2): 165-179.

19. Caballero, y C., E., R. R. Brenes y O. Jiménez-Quirós

1957. Helmintos de la República de Costa Rica. IV. Algunos tremátódos de animales domésticos y silvestres. Rev. Biol. Trop., 5 (2): 135-155.

20. Caballero, y C., E., y C. Díaz-Ungria

1958. Intento de un Catálogo de los Tremátodos Diegéneos registrados en Teerfís 
torio Venezolano. Mem. Soc. Cien. Nat. Salle, 18 (49): 19-36.

21. Castro, J. A.

1948. Fascioliasis hepática humana en Costa Rica. (Presentación de un caso). Rev. Kuba Med. Trop. y Parasitol., 4 (8): 160-161.

22. Castro, E. R., y H. Trenchi

1955. Fauns parasitológica comprobada en el Uruguı!y v bibliografía parasitológica nacional. Boletín $\mathrm{N}^{\circ} 1$ Ministerio de Ganadería y Agricultura. Uruguay.

23. CÉSPEDES, R.

1953. Ascaridiosis hepática y de las vías biliares. Estudio de cinco casos anatomoclínicos. Rev. Biol. Trop., 1 (2): 197-221.

24. Chauhan, B. S.

1953. Studies on the trematode fauna of India. Part. I. Subclase Monogenea. Rec. Indian Mus., 51: 113-208.

25. Chavarría, A. C

1937. Enfermedades parasitarias de los cerdos. Revista del Centro Nacional de Agricultura, 2(9-12): 430-436.

26. Chavarría, A. C.

1937. Parásitos internos y enfermedades parasitarias de los caballos en Costa Rica. Revista del Centro Nacional de Agricultura, 2 (9-12): 444-456.

27. Chavarría, A. C.

1939. Parásitos y enfermedades parasitarias del ganado vacuno en Costa Rica. Revista del Centro Nacional de Agricultura, 4(1-2): 25-32.

28. Chavarría, A. C.

1941. Parásitos y enfermedades parasitarias de las aves. Revista del Departamento Nacional de Agricultura, 6 (4-6): 98-113.

29. Chavarría, A. C.

1941. Parásitos de los animales domésticos. Departamento Nacional de Agricultura. Boletín técnico $\mathrm{N}^{\circ}$ 39. 48 pp. San Pedro, Montes de Oca, Costa Rica.

30. Chitwood, B. G., y M. B. Chitwood

1950. An Introduction to Nematology. Section I. Anatomy. VIII + 213 pp. Monumental Printing Co., Baltimore, $\mathrm{Md}$.

31. DAWES, B.

1956. The Trematoda, with special reference to British and other European forms. $\mathrm{XVI}+655$ pp. Univ. Press, Cambridge.

32. Dikmans, G.

1935. Parasites of cattle in Costa Rica. Proc. Helm. Soc. W ash., 2 (2): 83.

33. Fermosselle, B. I., y A. Portuondo del Pino

1935. Parasites of cattle in Costa Rica. Proc. Helm. Soc. IY'ash., 11 (3): 343.347.

34. Fermoselle, B. T., y A. Portuondo del Pino

1936. Hymenolepis diminuta. Caso humano. Rew. Parasitol. Clin. y Lab., Habana. 11 (4): 629-633. 
35. Flores-Barroeta, L., y E. Hidalgo

1960. Céstodos de Vertebrados. VII. Libro Jubilar Dr. Eduardo Caballero, pp. 357-376.

36. Flores-Barroeta, L., E. Hidalgo, y R. R. Brenes

1958. Céstodos de Vertebrados. IV. Rev. Biol. Trop., 6 (1): 55-78.

37. Flores-Barroeta, L., E. Hidalgo, y R. R. Brenes

1958. Céstodos de Vertebrados. VI. Rev. Biol. Trop., 6 (2): 167-188.

38. FuhrmanN, O.

1908. Die Cestoden der Vogel. Zool. Jabrb., Suppl., 10: 1-232.

39. FuhrmanN, O.

1932. Les tenias des oiseaux Mem. Inniv. Neuchatel, 8: 1-381.

40. HaRgIS, W. J., JR.

1959. Systematic Notes on the Monogenetic Trematodes. Proc. Helm. Soc. Wash., 26 (1): 14-31.

41. HubBs, C.

1853. Proposed modifications of the Stenzel system. Copeia, 4: 251-252.

42. JimÉnEZ-Quirós, O., y R. R. BRENES

1956. Presencia de Leiurus leptocephalus (Rudolphi, 1819) Leuckart, 1850 en Bradypus griseus griseus (Gray, 1871) Allen, 1891. Rev. Biol. Trop., 4 (2): $157-160$.

43. JimÉnez-Quirós, O., y R. R. BRENES

1957. Helmintos de la República de Costa Rica. V. Sobre la validez del género Controrchis Price, 1928 (Trematoda: Dicrocoeliidae) y descripción de Controrchis caballeroi, n. sp. Rev. Biol. Trop., 5 (1): 103-121.

44. Jiménez-Quirós, O., y R. R. BRENES

1958. Nota sobre el tremátodo Controrchis caballeroi Jiménez y Brenes, 1957. Rev. Biol. Trop, 6 (2): 189-190.

45. IIMÉNEZ-Quirós, O., y G. ARroyo

1960. Helmintos de la República de Costa Rica. XIV. Redescripción de Lutztrema obliquun Travassos, 1941, y descripción de Zonorchis macroovaricus n. sp. Rev. Biol. Trop., 8 (1): 53-61.

46. JimÉnEZ-Quirós, O., y G. ARROYo

1960. Helmintos de la República de Costa Rica. XV. Nematoda 3. Presencia de Litomosoides peñai n. sp. en Hemiderma perspicillatum aztecum (Saussure, 1860) Rev. Biol. Trop., 8 (1): 63-67.

47. Jiménez-Quirós, O., R. R. BRenes, y P. L. Vieto

1958. Parasitosis intestinal en el universitario costarricense I. Helmintiasis. Rev. Biol. Trop., 6 (1): 113-122.

48. JOYeux, C., y J. G. BAER

1936. Cestodes. Faune de France, 30: 1-613. Paris. 
49. LA Rue, G. R.

1957. Classification of digenetic Trematoda. A review and a new system. Exp. Parasii., 6 (3): 306-349.

50. LIESKe, H.

1954. Filariasis en Puerto Limón, Costa Rica. Rev. Biol. Trop., 2 (1): 37-44.

51. Manter, W. H.

1940. Digenetic trematodes of fishes from the Galapagos Islands and the neighboring Pacific. Allan Hancock Pacific Expeditions, 2 (14): 329-497.

52. MARín, T. E.

1955. Helmintiasis de los equinos de la zona atlántica. Tesis de Grado. IV + 150 pp. Universidad de Costa Rica.

53. Meserve, F. G.

1938. Some monogenetic trematodes from the Galapagos Islands and the neighboring Pacific. Allan Hancock Pacific Expeditions, 2 (5): 31-89.

54. Montero, C. R. E.

1944. Una nota sobre parasitismo intestinal de las cabras, y sus posibilidades de control. Tesis de Grado. 56 pp. Universidad de Costa Rica.

\section{Morera, P., y G. BArbareschi}

1956. Contribución al estudio de la toxoplasmosis experimental. Rev. Biol. Trop., 4 (2): 103-149.

56. Morgan, B. B., y P. A. Hawkins

1949. Veterinary Helminthology. IX +400 pp. Burges Publ. Co. Minneapolis, Minn.

57. Neveu-Lemaire, $M$.

1936. Traité D'Helminthologie Medicale et Veterinaire. xxiii +1514 pp. Vigot Frères, Paris.

58. Palombi, A.

1949. I Trematodi d'Italia. Parte I. Trematodi Monogenetici. Arch. Zool. Italiano., 34: 203-408.

59. Petrotschenko, V. I.

1956. Acantocéfalos de animales domésticos y silvestres. 1: 1-435. Isdatelstvo Akademii Nauk. SSSR. Moskva. (En ruso).

60. Petrotschenko, V. I.

1958. Acantocéfalos de animales domésticos y siluestres. 2: 1-458. Isdatelstvo Akademii Nauk. SSSR. Moskva. (En ruso).

61. Price, W. E.

1938. The monogenetic trematodes of Latin America. Livro Jubilar do Professor Lauro Travassos: pp. 407-413. Rio de Janeiro. Brasil. 
62. Quesada, S. W.

1939. Algunos parásitos de nuestro ganado de destace. Revista del Centro Nacional de Agricultura, 4 (7-8-9): 263-292.

63. Schapiro, L., y E. G. Nauck

1931. Observations on hookworn disease in Costa Rica based on post-mortem findings. Amer. J. Hyg., 14 (3): 705-714.

64. SKRJABIN, K. I.

1948. Trematody jivotnyj i cheloveka. Osnovy Trematodologii, 2: 1-600. Isdatelstvo Akademii Nauk. SSSR. Moskva. (En ruso).

65. SkrJabin, K. I.

1949. Trematody jivotnyj $i$ cheloveka. Osnovy Trematodologii, 3: 1-523. Isdatelstvo Akademii Nauk. SSSR. Moskva. (En ruso).

66. SKRJABIN, K. I.

1950. Trematody jivotnyj $i$ cheloveka. Osnovy Trematodologii, 4: 1-495. Isdatelstvo Akademii Nauk. SSSR. Moskva. (En ruso).

67. SKRJABIN, "K. I.

1951. Opredelitel paraziticheskij. Nematod. Oxyurata y Ascaridata, 2: 1-631. Isdatelstvo Akademii Nauk. SSSR. Moskva. (En ruso).

68. SKRJABIN, K. I.

1952. Opredelitel paraziticheskij. Nematod. Strongylata. 3: 1-890. Isdatelstvo Akademii Nauk. SSSR. Moskva. (En ruso).

69. SKRJABIN, K. I.

1952. Trematody jivotnyj $i$ cheloveka. Osnovy Trematodologii 7: 1.762. Isdatelstvo Akademii Nauk. SSSR. Moskva. (En ruso).

70. SKRJABIN, K. I.

1953. Osnovy Nematodologii. Parte I. 2: 1.351. Isdatelstvo Akademii Nauk. SSSR. Moskva. (En ruso).

71. SKrJabin, K. I.

1953. Osnovy Nematodologii. Parte II. 2: 1-616. Isdatelstvo Akademii Nauk. SSSR. Moskva. (En ruso).

72. SKRJABIN, K. I.

1953. Trematody jivotnyj $i$ cheloveka. Osnovy Trematodologii. 8: 1-618. Isdatelstvo Akademii Nauk. SSSR. Moskva. (En ruso).

73. Skrjabin, K. I.

1954. Osnovy Nematodologii. 3: 1-683. Isdatelstvo Akademii Nauk. SSSR. Moskva. (En ruso).

74. Skrjabin, K. I.

1954. Opredelitel Paraziticheskij. Nematod. Camallanata, Rhabditata, Tylenchata, Trichocephalata i Dioctophymata. 4: 1-997. Isdatelstvo Akademii Nauk. SSSR. Moskva. (En ruso). 
75. SKRJABIN, K. I.

1954. Osnovy Nematodologii. 4: 1-323. Isdatelstvo Akademi Nauk. SSSR. Moskva. (En ruso).

76. SKRJABIN, K. I.

1955. Osnovy Nematodologii. 5: 1-223. Isdatelstvo Akademii Nauk. SSSR. Moskva. (En ruso).

7\%. SkrJabin, K. I.

1955. Trematody jivotnyj $i$ cheloveka. Osnovy Trematodologii. 11: 1-751. Isdatelstvo Akademii Nauk. SSSR. Moskva. (En ruso).

78. SKRJABIN, K. I.

1956. Trematody jivotnyj $i$ cheloveka. Osnovy Trematodologii. 12: 1-932. Isdatelstvo Akademii Nauk. SSSR. Moskva. (En ruso).

79. SKrJabin, K. I.

1957. Osnovy Nematodologii, 6: 1-587. Isdatelstvo Akademii Nauk. SSSR. Moskva. (En ruso).

80. SKRJABin, K. I.

1958. Osnovy Nematodologii. 7: 1-424. Isdatelstvo Akademii Nauk. SSSR. Moskva. (En ruso).

81. SKRJABIN, K. I.

1958. Trematody jivotnyj $i$ cheloveka. Osnovy Trematodologii. 14: 1-934. Isdatelstro Akademii Nauk. SSSR. Moskva. (En ruso).

82. Spassky, A. A.

1951 Anoplocefaliati-Lentochni gelminti domashnib i dikib zbivotnyh. 1-735. Akademii Nauk. SSSR. Sokladi, Moskva. (En ruso).

83. Sproston, N. G.

1946. A Synopsis of the monogenetic trematodes. Trans. Zool. Soc. London, 25: 185-600.

84. Stiles, Ch. W., y A. Hassall

1920. Index-catalogue of medical veterinary zoology. Subjects: Roundworms. Bulletin (114): 1-886. Government Printing Office Washington.

85. Threkeld, W. L.

1958. Some nematode parasites of domestic animals. 338 pp. Economy Printing Co., Roanoke, Va. U. S. A.

86. T'ravassos, L.

1937. Revisăo da familia Trichostrongylidae Leiper, 1912. VII + 512 pp. Monografia $\mathrm{N}^{\circ}$ 1. Inst. Oswaldo Cruz, Rio de Janeiro.

87. Travassos, L.

1950. Introduçăo ao estudo da Helmintologia. Rev. Bíasil Biol., 1: 173.

88. Van Cleave, H. J.

1953. Acanthocephala of North American mammals. Illinois Biological Monographs. Vol. 23, Nos. 1-2: $x+$ 179. pp. Univ. of Illinois Press, Urbana. 
89. VARGAS, M.

1955. Contribución al estudio de Blattaria en Costa Rica. Tesis de Grado. 119 pp. Universidad de Costa Rica.

90. Víquez, S., C.

1935. Laboratorio de Química Biológica del Hospital San Juan de Dios. La Es. cuela Costarricense, (31-32): 74-81.

91. Vlquez, S., C.

1935. Animales renbnosos de Costa Rica. Parásitos intestinales de nuestres animales encontrados en sus investigasiones por el Licenciado Carlos Viquez Segreda. 313 pp. Imprenta Nacional. San José, Costa Rica.

92. Víquez, S., C.

1940. Nuestros Animales Venenusos. Parásitos de nuestros animales domésticos y de la selva. 240-299. Imprenta Nacional. San José, Costa Rica.

93. Vives, N., y R. ZeledóN

1957. Observaciones parasitológicas en ratas de San José. Rev. Biol. Trop., 5 (2): $173-194$

94. WARDLE, R. A., y J. A. MCLeOD

1952. The zoology of tapeworms xxiv + 780 pp. Univ. Minnesota Press. Minneapolis.

95. Yamagutr, S.

1958. Systema Helmintbum. The Digenetic Trematodes of Vertebrates. Vol. 1. Part II. xi +1575 pp. Interscience Publishers, N. Y., London.

96. Yorke, W., y P. A. Maplestone

1925. The nematode parasites of tertebrates. $x+536$ pp. J. \& A. Churchill, London.

97. Zeledón, R., y G. Arroyo

1960. Presencia de fornas larvirias de Oncicola oucicola (Acantocephala) en una gallina doméstica. Rev. Biol. Trop., 8(2): 197-199. 